\title{
Avifauna associada ao estuário do Saco da Fazenda, Itajai, Santa Catarina, Santa Catarina
}

\author{
Joaquim Olinto Branco ${ }^{1}$
}

\begin{abstract}
Avifauna associated to the estuary of Saco da Fazenda, Itajaí, Santa Catarina, Brazil. The estuary is an important feeding and resting site for mixed flocks of coastal seabirds, limnological inhabitants and also edge inhabitants. Once in a month, three census were done on the bird fauna with two hours intervals within observations, during the period from January/96 to December/98. The results showed the presence of 45 species, where $45,7 \%$ were regularly observed, while 17,9 occurred seasonally and $36,4 \%$ were occasional in the census. The highest abundance occurred during the winter and spring months, and the lowest during the summer and autumn. The medium annual diversity index varied between $1,68 \pm 0,36$ and $1,82 \pm 0,34$. The highest similarity of fauna was observed between the years 1997 and 1998.

KEY WORDS. Avifauna, census, seasonal changes, Santa Catarina
\end{abstract}

O estuário do Saco da Fazenda, apesar do impacto antrópico causado pelo afluxo de efluentes domésticos e resíduos sólidos, abriga uma grande diversidade de aves. O estuário atua como um importante local de alimentação e repouso para bandos mistos de aves marinhas costeiras, limícolas e habitantes das bordas.

Apesar dos $670 \mathrm{~km}$ de litoral e a existência de vários estuários, ao longo da costa, informações sobre aves associadas a ambientes de influência marítima em Santa Catarina, estão restrito aos trabalhos de BEGE \& PAULI (1988), SCHIEFLER \& SOARES (1994) e SOARES \& SCHIEFLER (1995).

Devido à carência de informações quali-quantitativas da avifauna estuarina e a intensa pressão antrópica sobre os estuários, este trabalho tem como objetivo fornecer subsídios básicos para o monitoramento da avifauna do estuário do Saco da Fazenda.

\section{MATERIAL E MÉTODOS}

O estuário do Saco da Fazenda está localizado entre as coordenadas

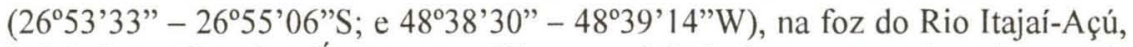
Itajaí, Santa Catarina. É um corpo d'água semi-fechado com uma área de aproximadamente $0,7 \mathrm{~km}^{2}$, resultante da ação antrópica que modificou a desembocadura original do rio com a construção dos moles de contenção. Apresenta substrato síltico-argiloso, profundidade máxima de dois metros, exceto nos canais de ligação com o rio, que atinge até nove metros e amplitude de maré inferior a 1,4 m. O estuário recebe aporte de água doce e efluentes domésticos do Ribeirão Schineider e bairro Saco da Fazenda.

1) Centro de Ciências Tecnológica, da Terra e do Mar, Universidade do Vale do Itajaí. Caixa Postal 360, 88301-970 Itajaí, Santa Catarina, Brasil.

E-mail: job@cttmar.univali.br. 
As aves, observadas pousadas sobre os moles, forrageando na água e nas margens desse estuário, foram monitoradas mensalmente, durante o período de janeiro/96 a dezembro/98.

Uma vez por mês, foram realizados três censos da avifauna, iniciando sempre às 8:00 h, com intervalo de duas horas entre as observações. O número médio de aves por mês, foi adotado como uma medida padrão de abundância (Tab. I).

De acordo com a ocorrência, as espécies foram agrupadas em três categorias: regular ( 9 a 12 meses), sazonal ( 6 a 8 meses) e ocasional ( 1 a 5 meses). O grau de similaridade da avifauna entre os anos de censos foram estimados através do índice de Jaccard (Southwood 1968). Com os valores médios de abundância, foram calculados o índice de diversidade de Shannon (LUDwIG \& REYNOLDS 1988), comparando-se a abundância mensal entre os anos de censos, através da análise de variância “one way" $(\mathrm{p}<0,05)$ (SOKAL \& ROHLF 1969).

Tab. I. Relação das espécies de aves e suas respectivas freqüências médias e desvio padrão (s) por ano de censo, no estuário do Saco da Fazenda, durante o período de 1996 a 1998. A ocorrência (oc) das espécies nos censos é representada por regular ( $r$ ), sazonal (s) e ocasional (o).

\begin{tabular}{|c|c|c|c|c|c|c|c|c|c|}
\hline \multirow{2}{*}{ Espécies } & \multicolumn{3}{|c|}{1996} & \multicolumn{3}{|c|}{1997} & \multicolumn{3}{|c|}{1998} \\
\hline & $N_{\text {Médo }}$ & s & $O C$ & $N_{\text {Médio }}$ & s & oc & $\mathrm{N}_{\text {Médio }}$ & s & oc \\
\hline \multicolumn{10}{|l|}{ Podicipedidae } \\
\hline $\begin{array}{l}\text { Podilymbus podiceps (Linnaeus, 1758) } \\
\text { Phalacrocoracidae }\end{array}$ & - & - & - & - & - & - & 0.6 & - & $\circ$ \\
\hline $\begin{array}{l}\text { Phalacrocorax brasilianus (Gmelin, 1789) } \\
\text { Fregatidae }\end{array}$ & 148,8 & 77.1 & $r$ & 133,8 & 114,5 & $r$ & 180,9 & 154,7 & r \\
\hline $\begin{array}{l}\text { Fregata magnificens Mathews, } 1914 \\
\text { Ardeidae }\end{array}$ & 6,1 & 5,0 & $r$ & 3,1 & 1,8 & s & 8,1 & 18,7 & s \\
\hline Ardea cocoi Linnaeus, 1766 & 1,3 & 0,3 & $r$ & 0,9 & 0,2 & $r$ & 0,9 & 0,3 & $r$ \\
\hline Casmerodius albus (Linnaeus, 1758) & 24,1 & 28,4 & $r$ & 36,6 & 39,3 & $r$ & 24,4 & 32,4 & $r$ \\
\hline Egretta thula (Molina, 1782) & 32,5 & 30,2 & $r$ & 38.1 & 28,8 & $r$ & 20,3 & 22,8 & $r$ \\
\hline Egretta caerulea (Linnaeus, 1758) & 4,4 & 4,2 & $r$ & 12,7 & 9,2 & $r$ & 4,4 & 3,5 & $r$ \\
\hline Bubulcus ibis (Linnaeus, 1758) & 2,0 & - & 0 & - & - & - & - & - & - \\
\hline Butorides striatus (Linnaeus, 1758) & 3,2 & 2,1 & $r$ & 2,6 & 1,0 & $r$ & 2,1 & 0,8 & $r$ \\
\hline Syrigma sibilatrix (Temminck, 1824) & 1,0 & - & 0 & - & - & - & - & - & - \\
\hline Nycticorax nycticorax (Linnaeus, 1758) & 1,4 & 0,8 & $r$ & 1,5 & 0,6 & $r$ & 1,9 & 0,4 & $r$ \\
\hline Nyctanassa violacea (Linnaeus, 1758) & 2,4 & 1,1 & $r$ & 2,4 & 0,6 & $r$ & 3,1 & 0,8 & $r$ \\
\hline Threskiornithidae & & & & & & & & & \\
\hline $\begin{array}{l}\text { Platalea ajaja Linnaeus, } 1758 \\
\text { Cathartidae }\end{array}$ & 2,1 & 0,7 & s & 1,7 & 0,7 & s & 2,1 & 1,3 & 0 \\
\hline Coragyps atratus (Bechstein, 1793) & 23,9 & 21,0 & $r$ & 28,3 & 25,8 & $r$ & 33,7 & 52,4 & $r$ \\
\hline Cathartes aura (Linnaeus, 1758) & - & - & - & - & - & - & 0,6 & - & o \\
\hline Anatidae & & & & & & & & & \\
\hline Anas sp. & 2,0 & - & 0 & - & - & - & - & - & - \\
\hline $\begin{array}{l}\text { Amazonetta brasiliensis (Gmelin, 1789) } \\
\text { Accipitridae }\end{array}$ & - & - & - & 2,8 & 2,5 & 0 & 2,9 & 2,0 & 0 \\
\hline $\begin{array}{l}\text { Leucopternis lacemulata (Temminck, 1827) } \\
\text { Falconidae }\end{array}$ & - & - & - & 1,0 & - & 0 & - & - & - \\
\hline Milvago chimachima (Vieillot, 1816) & 0,9 & 0,3 & $\mathrm{~s}$ & 1,2 & 0,4 & s & 0,3 & - & 0 \\
\hline Milvago chimango (Vieillot, 1816) & 1,0 & - & 0 & 1,0 & - & 0 & - & - & - \\
\hline Polyborus plancus (Miller, 1777) & 2,0 & - & 0 & 1,5 & 0,7 & 0 & 0,7 & 0,6 & s \\
\hline Rallidae & & & & & & & & & \\
\hline Rallus sanguinolentus Swainson, 1837 & 1,0 & - & 0 & - & - & - & - & - & - \\
\hline Aramides cajanea (Mueller, 1776) & 1,0 & - & 0 & - & - & - & - & - & - \\
\hline Gallinula chloropus (Linnaeus, 1758) & 12,1 & 11,2 & r & 16.7 & 12,5 & $r$ & 9,2 & 6,0 & $r$ \\
\hline $\begin{array}{l}\text { Fulica armillata Vieillot, } 1817 \\
\text { Jacanidae }\end{array}$ & 10,0 & - & 0 & - & - & - & - & - & - \\
\hline Jacana jacana (Linnaeus, 1766) & - & - & - & 1,1 & 1,2 & 0 & 0,3 & Co & $\begin{array}{l}\circ \\
\text { inue }\end{array}$ \\
\hline
\end{tabular}


Tab. I. Continuação.

\begin{tabular}{|c|c|c|c|c|c|c|c|c|c|}
\hline \multirow{2}{*}{ Espécies } & \multicolumn{3}{|c|}{1996} & \multicolumn{3}{|c|}{1997} & \multicolumn{3}{|c|}{1998} \\
\hline & $N_{\text {Médio }}$ & s & OC & $N_{\text {Médio }}$ & s & oc & $N_{\text {Médio }}$ & s & oc \\
\hline Haematopodidae & & & & & & & & & \\
\hline $\begin{array}{l}\text { Haematopus palliatus Temminck, } 1820 \\
\text { Charadriidae }\end{array}$ & - & - & - & - & - & - & 0,6 & - & o \\
\hline Vanellus chilensis (Molina, 1782) & 7,5 & 3,4 & $r$ & 11,3 & 5,9 & $r$ & 9,2 & 1,5 & $r$ \\
\hline Charadrius semipalmatus Bonaparte, 1825 & 5,8 & 4,6 & s & 6,7 & 4,9 & $r$ & 7,3 & 2,8 & s \\
\hline $\begin{array}{l}\text { Charadrius collaris Vieillot, } 1818 \\
\text { Scolopacidae }\end{array}$ & 1,4 & 0,8 & 0 & 2,6 & 1,3 & ० & 3,3 & - & 0 \\
\hline Tringa flavipes (Gmelin, 1789) & 1,0 & - & 0 & - & - & - & - & - & - \\
\hline $\begin{array}{l}\text { Actitis macularia (Linnaeus, 1766) } \\
\text { Laridae }\end{array}$ & 5,0 & 3,1 & s & 5,9 & 7,0 & $r$ & 3,4 & 1,0 & s \\
\hline Larus dominicanus Lichtenstein, 1823 & 148,4 & 64,2 & $r$ & 152,1 & 33,8 & $r$ & 178,9 & 87,6 & $r$ \\
\hline Larus maculipennis Lichtenstein, 1823 & 2,7 & 0,7 & ○ & 10,1 & 13,4 & 0 & 2,8 & 2,6 & 0 \\
\hline Sterna hirundinacea Lesson, 1831 & 5,8 & 4,5 & 0 & 3,8 & 1,9 & 0 & - & - & - \\
\hline Sterna maxima Boddaert, 1783 & 10,6 & 6,5 & s & 10,3 & 10,0 & s & 9,5 & 7,3 & s \\
\hline $\begin{array}{l}\text { Sterna eurygnatha Saunders, } 1876 \\
\text { Rynchopidae }\end{array}$ & 19,9 & 31,9 & s & 27,3 & 59,4 & $r$ & 12,6 & 16,5 & s \\
\hline $\begin{array}{l}\text { Rynchops niger Linnaeus, } 1758 \\
\text { Cuculidae }\end{array}$ & 1,7 & 1,7 & s & 1,0 & 0,5 & 0 & 0,3 & - & 0 \\
\hline Crotophaga ani Linnaeus, 1758 & 4,0 & 1,1 & 0 & 4,4 & 2,1 & 0 & 4,0 & - & 0 \\
\hline $\begin{array}{l}\text { Guira guira (Gmelin, 1788) } \\
\text { Alcedinidae }\end{array}$ & 3,5 & 2,7 & s & 5,3 & - & 0 & 3,0 & 2,0 & o \\
\hline Ceryle torquata (Linnaeus, 1766) & 0,9 & 0,4 & $r$ & 0,8 & 0,2 & s & 0,6 & 0,2 & 0 \\
\hline $\begin{array}{l}\text { Chloroceryle americana (Gmelin, 1788) } \\
\text { Furnariidae }\end{array}$ & 1,0 & - & 0 & - & - & - & - & - & - \\
\hline $\begin{array}{l}\text { Furnarius rufus (Gmelin, 1788) } \\
\text { Tyrannidae / Tyranninae }\end{array}$ & 7,2 & 2,9 & $r$ & 7,8 & 2,4 & $r$ & 7,6 & 2,3 & $r$ \\
\hline $\begin{array}{l}\text { Pitangus sulphuratus (Linnaeus, 1766) } \\
\text { Troglodytidae }\end{array}$ & 6,3 & 2,0 & $r$ & 6,4 & 1,8 & $\mathrm{r}$ & 7,4 & 1,7 & $r$ \\
\hline Troglodytes aedon Vieillot, 1807 & 5,3 & 2,3 & $r$ & 8,0 & 4,1 & $r$ & 7,1 & 1,8 & $r$ \\
\hline
\end{tabular}

\section{RESULTADOS}

\section{Composição da avifauna}

Durante o período de 1996 a 1998 foram realizados um total de 108 censos, sendo efetuados 36 por ano e registrado a ocorrência de 45 espécies de aves no estuário do Saco da Fazenda (Tab. I).

Em 1996 foi constatada a presença de 39 espécies, 33 gêneros e 17 famílias (Tab. I). Dessas espécies, 17 apresentaram ocorrência regular, 8 sazonal e 14 espécies ocasional (Tab. I). No ano de 1997, houve uma redução de espécies, permanecendo em 34 espécies, 28 gêneros e 18 famílias; sendo que 18 espécies foram regulares nos censos, 5 sazonais e 13 ocasionais (Tab. I). Em 1998 ocorreu padrão semelhante à 1997, com 34 espécies, 30 gêneros e 19 famílias; destas, 15 espécies apresentaram ocorrência regular, 7 sazonal e 42 foram ocasionais (Tab. I).

Em geral, $45,7 \%$ das espécies foram regularmente avistadas, enquanto que $17,9 \%$ ocorreram sazonalmente e $36,4 \%$ apresentaram ocorrência ocasional nos censos (Fig. 1a).

O número de espécies por mês de censo oscilou moderadamente ao longo do período de estudo, sendo que a maior e a menor ocorrência de espécies no ano de 1996, foi registrada, respectivamente nos meses de outubro (28) e junho-novembro (21); em 1997, nos meses de julho (26) e dezembro (14); enquanto que no ano de 1998, foram registradas em abril-maio (23) e dezembro (15) (Fig. Ib). 
A análise da curva acumulada de espécies, indica um aumento gradual entre janeiro a maio/96, seguido de breve pausa, com incremento a partir de julho/96 e intercalando períodos de estabilidade com a incorporação de novas espécies, até alcançar a estabilização em julho/98 (Fig. 1b).
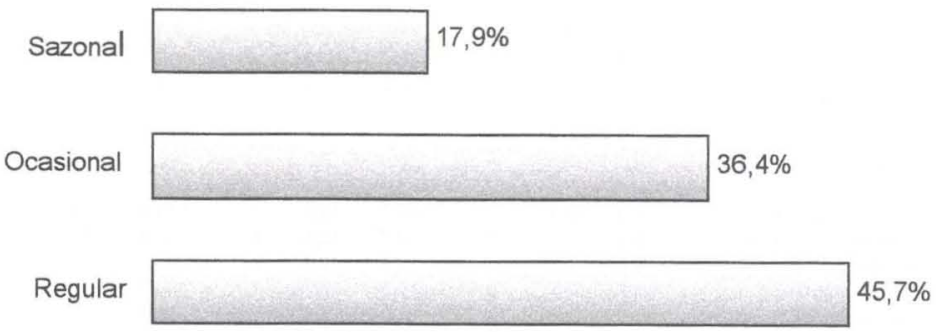

a

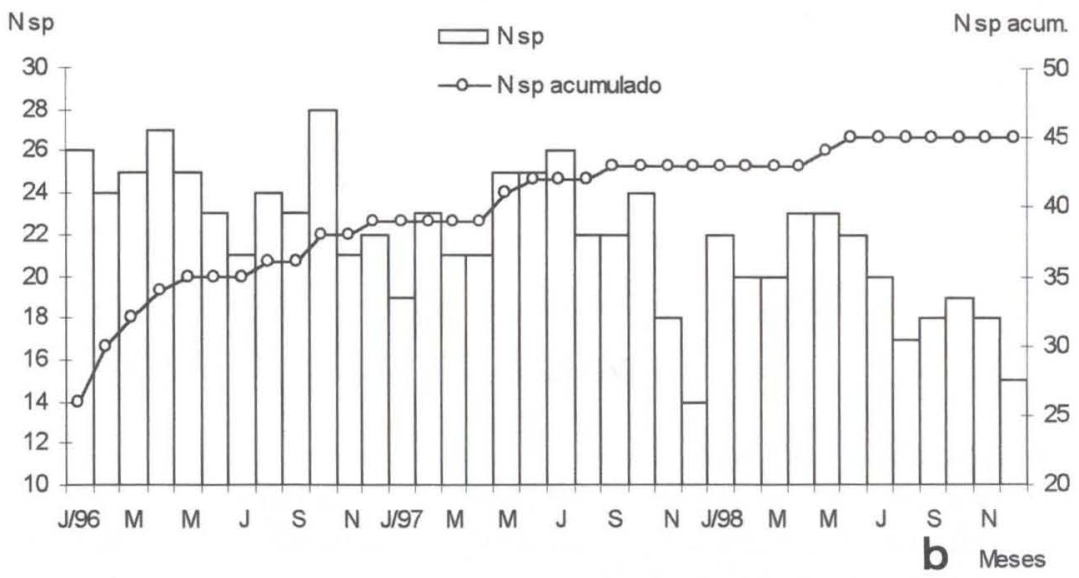

Fig. 1. Ocorrência relativa das categorias de espécies (a); variação mensal do número de espécies e do número acumulado de espécies (b) entre 1996 a 1998.

\section{Abundância nos censos}

A figura 2, indica que ocorreram flutuações sazonais na abundância média da avifauna do estuário do Saco da Fazenda. De maneira geral, as maiores abundâncias ocorreram durante os meses de inverno e primavera, enquanto que as menores, foram observadas entre os meses de verão e outono (Fig. 2). Embora com abundância relativamente diferente entre os meses de censo, a ANOVA ( $\mathrm{F}=0,2395 ; \mathrm{gl} 2-33$ ) não apresentou diferença significativa entre os anos de estudo.

O número total médio de aves registradas durante o ano de 1996 foi de 5596,7 exemplares, com média mensal de 466,3 aves (Fig. 2a); em 1997, houve um pequeno incremento, ficando em torno de 5897,5 aves e uma abundância média mensal de 491,4 indivíduos (Fig. 2b); no ano 1998, chegou aos 6176,9 exemplares e uma média mensal de 514,7 aves (Fig. 2c). 

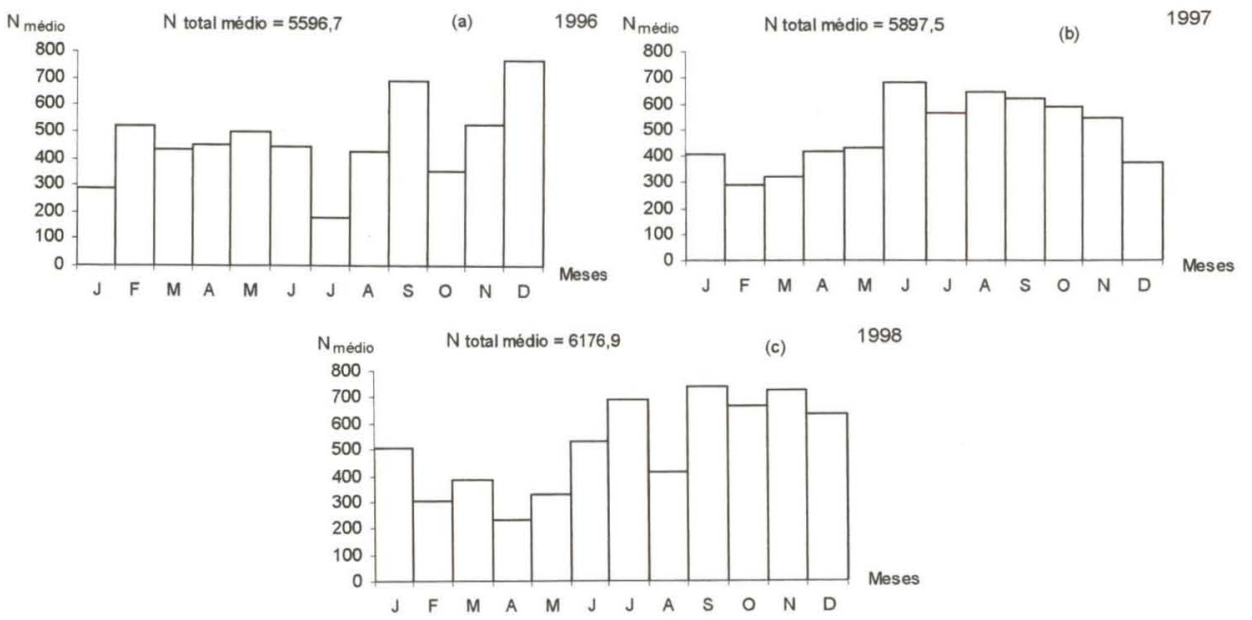

Fig. 2. Abundância média mensal em número de exemplares, durante o período de 1996 (a), 1997 (b), 1998 (c).

A figura 3 mostra a contribuição relativa em número médio das espécies de ocorrência regular mais abundantes nos censos. Destas, o biguá Phalacrocorax brasilianus (Gmelin, 1789) e o gaivotão Larus dominicanus Lichtenstein, 1823 contribuíram com as maiores frequências, seguidas pelas garças Casmerodius albus (Linnaeus, 1758), Egretta thula (Molina, 1782), pelo urubu Coragyps atratus (Bechstein, 1793) e o frango d'água Gallinula chloropus (Linnaeus, 1758) (Fig. 3a, b, c).

1996

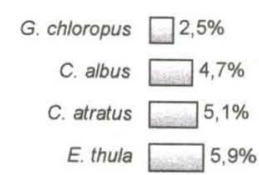

L. dominicanus

P. brasilianus

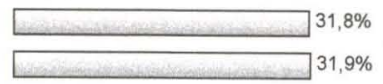

(a)

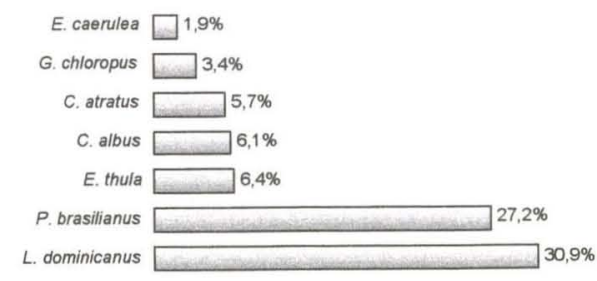

1998

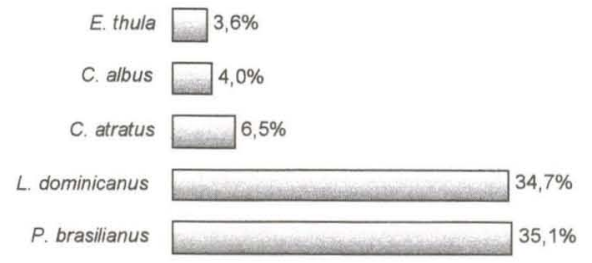

Fig. 3. Contribuição relativa das espécies regulares mais abundantes, em número médio de exemplares, no periodo de 1996 (a), 1997 (b), 1998 (c).

Revta bras. Zool. 17 (2): 387 - 394, 2000 


\section{Diversidade entre os anos}

O índice de diversidade de Shannon (H'), apresentou flutuações moderadas ao longo dos anos de estudo (Fig. 4). Em 1996, a maior diversidade foi registrada em julho $(2,29)$ e a menor em setembro $(0,36)$; a média anual foi de $1,78 \pm 0,53$ (Fig. 4a). Em 1997, a maior diversidade ocorreu em junho $(2,31)$ e a menor em dezembro $(1,17)$, com diversidade média anual de 1,82 $\pm 0,34$ (Fig. 4b). No ano de 1998, a maior diversidade ocorreu em abril $(2,29)$ e a menor em dezembro $(1,13)$, com média anual de 1,68 $\pm 0,36$ (Fig. 4c). Não foram observadas diferenças significativas ao longo do ano entre o índice de diversidade e os anos de censos ( $\mathrm{F}=0,3584$; gl 2-33).
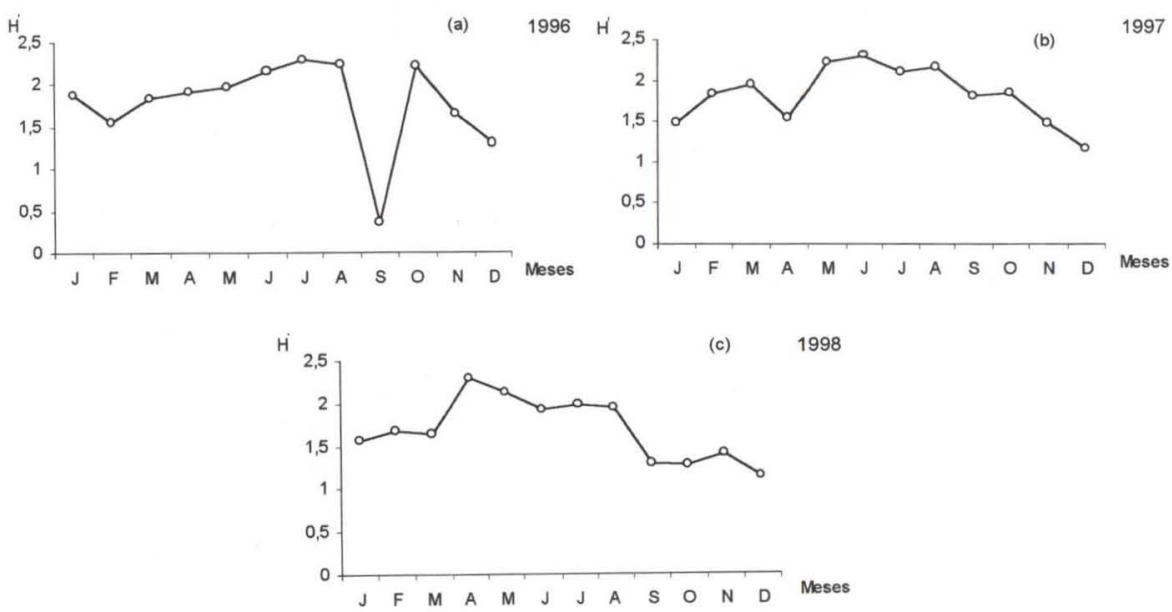

Fig. 4. Variação mensal do índice de diversidade de Shannon (H'), nos anos de 1996 (a), 1997 (b), 1998 (c).

\section{Similaridade entre os anos}

$\mathrm{O}$ índice de Jaccard apresentou similaridade elevada entre os anos de censo, com valores acima de $65 \%$, sendo os maiores valores observados entre os anos de 1997 e 1998 (Fig. 5).

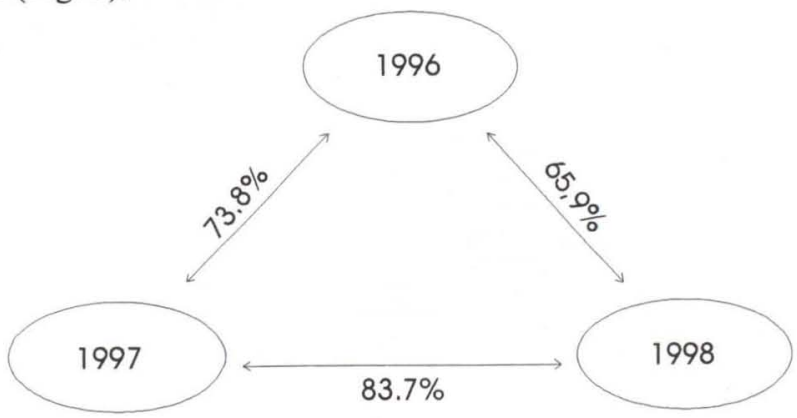

Fig. 5. Similaridade da avifauna entre os anos de censos no estuário do Saco da Fazenda. 


\section{DISCUSSÃO}

Do total de espécies registradas para o estuário do Saco da Fazenda, 64,4\% das espécies observadas foram comuns aos três anos de estudo, 17,8\% foram exclusivas de 1996; enquanto que em 1997, apenas 2,2\% e 6,7\% em 1998. Quatro espécies (8,9\%) ocorreram, somente nos anos de 1997 e 1998.

SCHIEFLER \& SOARES (1994), estudando a avifauna da Praia de Navegantes, Santa Catarina, distante cerca de 200 metros do estuário, registraram a ocorrência de 27 espécies, sendo que 20 espécies foram comuns aos dois ambientes. Das 59 espécies de aves associadas à ecossistemas de influência marítima do litoral do Paraná (MORAES \& KRUL 1995), 31 foram observadas no estuário do Saco da Fazenda. Assim como, das 16 espécies de aves que ocorrem na Ilha da Galheta, Laguna, Santa Catarina, (SOARES \& SCHIEFLER 1995), 12 podem ser encontradas na área de estudo.

As aves marinhas costeiras pertencentes à família Laridae e as limícolas das famílias Phalacrocoracidae e Ardeidae foram responsáveis pelas maiores abundâncias ao longo dos anos de censo; enquanto que as habitantes das bordas do estuário apresentaram as menores abundâncias. OLMOS (1989), trabalhando com a avifauna da baixada do pólo industrial de Cubatão, São Paulo, constatou que metade das espécies observadas apresentaram hábitos palustres.

Das 45 espécies registradas no estuário do Saco da Fazenda, apenas 36,4\% delas apresentaram ocorrência ocasional nos censos, enquanto que as regulares representaram $45,7 \%$ e as sazonais $17,9 \%$. As flutuações observadas na abundância média da avifauna do estuário podem ser atribuídas, em parte, aos eventos do ciclo de vida de algumas espécies. Assim, nos meses de inverno, pode ser observado um incremento significativo na abundância das garças $C$. albus e E. thula, bem como caracteriza a chegada de Larus maculipennis Lichtenstein, 1823, seguida da redução do número de biguás $P$. brasilianus e deslocamento dos adultos do gaivotão $L$. dominicanus para as áreas de reprodução. Na primavera, observa-se uma redução abrupta na abundância das garças, retorno de L. maculipennis para as áreas de origem e aumento considerável na abundância de biguás. Embora com o retorno dos adultos de $L$. dominicanus e o recrutamento dos juvenis, no final da primavera, acrescido da permanência dos biguás no estuário, a abundância média da avifauna atinge as menores taxas durante os meses de verão e outono.

O índice de diversidade de Shannon, largamente utilizado nos estudos de comunidades, parece não ser freqüentemente utilizado nos trabalhos de avifauna de locais alagados. Apesar disso, os valores mensais obtidos no estuário do Saco da Fazenda oscilaram entre 0,36 a 2,31, sendo que a média anual ficou entre 1,68 a 1,82. SCHIEFLER \& SOARES (1994) utilizando o índice de diversidade de Simpson, obtiveram uma diversidade anual para a avifauna da Praia de Navegantes, Santa Catarina, da ordem de 0,910 . O índice de similaridade de Jaccard, demonstrou uma afinidade acima de $65 \%$ entre os anos de censos. Essa similaridade é diretamente influenciada pelas flutuações sazonais das populações do estuário.

O estuário do Saco da Fazenda, apesar do impacto antrópico causado pelo afluxo de efluentes domésticos do Ribeirão Schineider, bairro do Saco da Fazenda e deposição de resíduos sólidos, carreados pelo Rio Itajaí-Açú; abriga uma grande 
diversidade de aves. O estuário atua como um importante local de alimentação e repouso para bandos mistos de aves marinhas costeiras, como L. dominicanus e os esternídeos (Sterna eurygnatha Saunders, 1876, S. maxima Boddaert, 1783 e S. hirundinacea Lesson, 1831); aves limícolas como P. brasilianus, ardeídeos [C. albus, Egretta thula e E. caerulea (Linnaeus, 1758)], Charadrius semipalmatus Bonaparte, 1825 e C. collaris Vieillot, 1818, Gallinula chloropus. Atua ainda, como uma fonte alternativa de alimento para habitantes das bordas, como Vanellus chilensis (Molina, 1782), Pitangus sulphuratus (Linnaeus, 1766) que durante a baixamar excursionam pela planície de maré à procura de poliquetas e crustáceos.

AGRADECIMENTOS. Aos colegas do CTTMar, Professores: José Gustavo Natorf de Abreu, Maria Inês Santos e Carlos Augusto F. Schettini, pela utilização de dados não publicados e auxílio na determinação da área do Estuário do Saco da Fazenda. Ao Dr. José Fernando Pacheco e um dos revisores anônimos pelas sugestões e correções.

\section{REFERÊNCIAS BIBLIOGRÁFICAS}

Bege, L.A.R. \& B.T. PaUli. 1988. As aves nas Ilhas Moleques do Sul - Santa Catarina: Aspectos da ecologia, etologia e anilhamento de aves marinhas. Florianópolis, FATMA, 64p.

LUDWIG, J.A. \& J.F. REYNOLDS. 1988. Statistical ecology: a primer on methods and computing. New York, John Wiley \& Sons Inc., 338p.

MoRAES, V.S. \& R. KRUL. 1995. Aves associadas a ecossistemas de influência marítima no litoral do Paraná. Arq. Biol. Tecnol., Curitiba, 38 (1): 121-134.

Olmos, F. 1989. A avifauna da baixada do pólo industrial de Cubatão. Rev. Brasil. Biol., Rio de Janeiro, 49 (2): 373-379.

SCHIEFI.ER, A.F. \& M. SOARES. 1994. Estudo comparativo da avifauna das praias de Navegantes e Laguna, Santa Catarina. Biotemas, Florianópolis, 7 (1/2): 31-45.

SoAres, M. \& A.F. SCHIEFLER. 1995. Aves da Ilhota da Galheta, Laguna, SC, Brasil. Arq. Biol. Tecnol., Curitiba, 38 (4): 1101-1107.

SOKAL, R.R. \& F.J. RoIILF. 1969. Biometry, the principles and practies of statistics in biological research. San Francisco, W.H. Freeman and Co., 776p.

Southwood, T.R.E. 1968. Ecological methods. London, Chapman and Holl, 368p.

Recebido em 27.IX.1999; aceito em 14.IV.2000

Revta bras. Zool. 17 (2): 387 - 394, 2000 